\title{
SOME NEW EVIDENCE FOR THE FONTAINE-MAZUR CONJECTURE
}

\author{
Christian MAIre
}

\begin{abstract}
In this paper, thanks to the work of M. Lazard, we obtain some new evidence
\end{abstract} for the conjecture of Fontaine-Mazur in the spirit of Boston's results.

Let $\mathbf{K}$ be a number field, $p$ a prime number and $S$ a finite set of places of $\mathbf{K}$. Assume that $S$ is prime to $p$ : for every finite place $v \in S$, the absolute norm of $v$ is prime to $p$. Denote by $\mathbf{K}_{S}$ the maximal pro-p-extension of $\mathbf{K}$ unramified outside $S$ and put $\mathcal{G}_{S}=\operatorname{Gal}\left(\mathbf{K}_{S} / \mathbf{K}\right)$.

A consequence of a conjecture of Fontaine and Mazur [5] asserts that there is no infinite analytic quotient of $\mathcal{G}_{S}$. The philosophy of this conjecture is that every analytic quotient of $\mathcal{G}_{S}$ should come from geometry, and then, thanks to the above conditions on $S$, this quotient should be finite.

The study of analytic pro-p-groups was initiated by Michel Lazard in a famous paper [9]. In particular, in this paper, he describes relationships between various important invariants of these groups such as: the dimension of the group $\mathcal{G}$ as $\mathbb{Q}_{p}$-manifold, the structure of the Lie algebra of $\mathcal{G}$, and the virtual cohomological dimension of $\mathcal{G}$.

Using some of these tools, Boston in [1] and in [2] gives for the first time arithmetical criteria for verifying special cases of Fontaine-Mazur conjecture. In [12], in the same spirit, Wingberg considers the CM-case. Here, one obtains:

Theorem 1. Let $\mathbf{K} / \mathbf{F}$ be an extension of number fields with finite group $\Delta$ of order prime to $p$. Let $\mathbf{L} / \mathbf{K}$ be a Galois pro-p-extension with group $\mathcal{G}$ such that: (i) $\mathbf{L} \subset \mathbf{K}_{S}$; (ii) $\mathbf{L} / \mathbf{F}$ is Galois. Put $\mathcal{G}_{1}=\mathcal{G} / \mathcal{G}^{p}[\mathcal{G}, \mathcal{G}]$.

If the $\Delta$-representation of $\bigwedge^{2}\left(\mathcal{G}_{1}\right)$ does not contain the $\Delta$-representation of $\mathcal{G}_{1}$, then $\mathcal{G}$ is not uniformly powerful.

From this result, we deduce some new evidence for the Fontaine-Mazur conjecture in the non-abelian case and in the cyclic case.

For the CM-case, by using a result of Gras [6], one obtains a new proof of a result of Wingberg [12]:

Received by the editors July 31, 2005.

2000 Mathematics Subject Classification. Primary 11R37; Secondary 12G10 .

Key words and phrases. pro- $p$-extensions, analytic pro- $p$-groups, conjecture of Fontaine-Mazur. 
Corollary 2. Assume $p>2$. Let $\mathbf{K}$ be a CM-field with maximal totally real subfield $\mathbf{F}$, and let $d\left(\mathcal{G}_{\emptyset}\right)=d^{+}+d^{-}$be the decomposition of the generator rank of $\mathcal{G}_{\emptyset}$ under the action of $\operatorname{Gal}(\mathbf{K} / \mathbf{F})$. Put $\delta_{p}=1$ if $\mathbf{K}$ contains the pth roots of unity, 0 otherwise. If $d^{+} d^{-}>d^{-}+\delta_{p}$, then $\mathcal{G}_{\emptyset}$ is not uniformly powerful.

\section{Uniformly powerful pro- $p$-groups}

Definition 3. A pro-p-group $\mathcal{G}$ is called powerful if the quotient $\mathcal{G} / \overline{\mathcal{G}^{p}}$ (for $p=2$, $\mathcal{G}\left(\overline{\mathcal{G}^{4}}\right)$ is abelian.

Before defining the notion of uniformly powerful pro-p-group, let us give some more notation. For a pro- $p$-group $\mathcal{G}$, define $P_{i}(\mathcal{G})$ to be its central descending series:

$$
P_{1}(\mathcal{G})=\mathcal{G}, \quad P_{i+1}(\mathcal{G})=P_{i}(\mathcal{G})^{p}\left[\mathcal{G}, P_{i}(\mathcal{G})\right] .
$$

In what follows, we will denote by $\mathcal{G}_{1}$ the quotient $\mathcal{G} / \mathcal{G}^{p}[\mathcal{G}, \mathcal{G}]=\mathcal{G} / P_{2}(\mathcal{G})$ and by $d(\mathcal{G})$ the $p$-rank of $\mathcal{G}$, that is the dimension of $\mathcal{G}_{1}$ over $\mathbb{F}_{p}$.

Definition 4. A finitely generated pro-p-group $\mathcal{G}$ is called uniformly powerful if

(1) $\mathcal{G}$ is powerful

(2) for all $i, \# P_{i}(\mathcal{G}) / P_{i+1}(\mathcal{G})=\# \mathcal{G} / P_{2}(\mathcal{G})$.

Note that, as shown in [3], a finitely generated powerful pro-p-group $\mathcal{G}$ is uniformly powerful if and only if $\mathcal{G}$ is torsion free.

Definition 5. A topological group $\mathcal{G}$ is p-adic analytic if $\mathcal{G}$ has a structure of a p-adic manifold such that the map $\mathcal{G} \times \mathcal{G} \rightarrow \mathcal{G}:(x, y) \mapsto x y^{-1}$ is analytic.

As explained in [3], Chapter 8 , a uniformly powerful pro-p-group $\mathcal{G}$ is analytic. But in fact:

Theorem 6 ([3], Theorem 8.32). Let $\mathcal{G}$ be an analytic pro-p-group. Then $\mathcal{G}$ admits an open uniformly powerful sub-group $\mathfrak{U}$.

As noted by the authors of [3], the class of uniformly powerful pro-p-groups is in the class of "groupes $p$-saturables", following Lazard's terminology. The great interest of the groups of the last class is that their cohomology is the same as the cohomology of their Lie algebra (see [9], chapter V). 
Theorem 7 (Lazard [9]). Let $\mathcal{G}$ be a uniformy powerful pro-p-group. Then, for $i \geq 1$

$$
H^{i}\left(\mathcal{G}, \mathbb{F}_{p}\right) \simeq \bigwedge^{i}\left(\mathcal{G}_{1}^{*}\right),
$$

where $\bigwedge^{i}\left(\mathcal{G}_{1}^{*}\right)$ denotes the $i$-th exterior power of the dual $\mathcal{G}_{1}^{*}$ of $\mathcal{G}_{1}$.

One can find a proof of this in [11]. Note that if $\mathcal{G}$ is uniformly powerful, its cohomological dimension is exactly its $p$-rank.

Now one can follow the strategy of Boston. Suppose that we have the following exact sequence of Galois groups:

$$
1 \longrightarrow \mathcal{G} \longrightarrow \Gamma \longrightarrow \Delta \longrightarrow 1,
$$

where $\Delta$ is a finite group of order prime to $p$ and $\mathcal{G}$ is uniformly powerful. The group $\Delta$ acts on the groups $H^{i}\left(\mathcal{G}, \mathbb{F}_{p}\right)$. Hence thanks to Theorem 7 , one can compute exactly the characters of the $\Delta$-module $H^{2}\left(\mathcal{G}, \mathbb{F}_{p}\right)$. Comparing this with the arithmetic of $\mathcal{G}$, one obtains in some cases a contradiction.

\section{Theorem 1 and its consequences}

2.1. Basic properties on representations. First recall some well-know properties about representations in the semi-simple case (see [10], chapters 14, 15 and 16).

Let $\Delta$ be a finite group of order prime to $p$. In this situtation, every finitely generated $\mathbb{F}_{p}[\Delta]$-module $M$ is projective. Then, to each such $M$, one can associate a unique (up to isomorphism) $\mathbb{Z}_{p}[\Delta]$-projective module $N$ such that $N / p N$ is isomorphic to $M$. Now one has to note that two $\mathbb{Z}_{p}[\Delta]$-projective modules $N$ and $N^{\prime}$ are isomorphic if and only if $\mathbb{Q}_{p} \otimes N$ and $\mathbb{Q}_{p} \otimes N^{\prime}$ are $\Delta$-isomorphic. Hence, taking an $\mathbb{F}_{p}[\Delta]$-module $M$, to study the action of $\Delta$ on it is equivalent to look at the action of $\Delta$ on $\mathbb{Q}_{p} \otimes N$ and then finally to find the decomposition of the character of $\mathbb{Q}_{p} \otimes N$ in terms of absolutely irreducible characters $\varphi$. In other words, the irreducible representations of $\Delta$ over $\mathbb{F}_{p}$ are in bijection with the irreducible representations of $\Delta$ over $\mathbb{Q}_{p}$.

Next, if $M$ is an $\mathbb{F}_{p}[\Delta]$-module, we will identify $M$ with its associated $\mathbb{Q}_{p}[\Delta]$-module $N$. In particular, if $M$ is an $\mathbb{F}_{p}[\Delta]$-module, we will denote by

$$
\chi(M):=\chi(N)=\sum_{\varphi} a_{\varphi} \varphi
$$

the decomposition of the character $\chi(N)$ in terms of absolutely irreducible characters $\varphi$, where $N$ is the associated $\mathbb{Q}_{p}[\Delta]$-module of $M$.

To finish this part, we recall a classical convention. Let $N$ and $N^{\prime}$ be two $\mathbb{Q}_{p}[\Delta]$ modules and let $\chi(N)=\sum_{\varphi} a_{\varphi} \varphi$ and $\chi\left(N^{\prime}\right)=\sum_{\varphi} a_{\varphi}^{\prime} \varphi$ be the decomposition of the characters of these modules into absolutely irreducible characters $\varphi$. Then $\chi(N) \leq$ $\chi\left(N^{\prime}\right)$ means that for every absolutely irreducible character $\varphi, a_{\varphi} \leq a_{\varphi}^{\prime}$, and $\chi(N)<$ $\chi\left(N^{\prime}\right)$ means that $\chi(N) \leq \chi\left(N^{\prime}\right)$ and there exists $\varphi$ such that $a_{\varphi}<a_{\varphi}^{\prime}$. 
2.2. The proof of Theorem 1 . The exact sequence of $\mathcal{G}$-modules

$$
1 \longrightarrow \mathbb{F}_{p} \longrightarrow \mathbb{Q}_{p} / \mathbb{Z}_{p} \longrightarrow \mathbb{Q}_{p} / \mathbb{Z}_{p} \longrightarrow 1
$$

gives

$$
\cdots \longrightarrow H^{1}\left(\mathcal{G}, \mathbb{Q}_{p} / \mathbb{Z}_{p}\right) \stackrel{p}{\longrightarrow} H^{1}\left(\mathcal{G}, \mathbb{Q}_{p} / \mathbb{Z}_{p}\right) \longrightarrow H^{2}\left(\mathcal{G}, \mathbb{F}_{p}\right) \longrightarrow \cdots
$$

By passing to the dual, one obtains:

$$
H^{2}\left(\mathcal{G}, \mathbb{F}_{p}\right)^{*} \rightarrow \mathcal{G}^{a b}[p] .
$$

Now if the group $\mathcal{G}$ is uniformly powerful, the $\Delta$-module $H^{2}\left(\mathcal{G}, \mathbb{F}_{p}\right)$ is exactly $\bigwedge^{2}\left(\mathcal{G}_{1}{ }^{*}\right)$. Hence, as $\Delta$-module:

$$
H^{2}\left(\mathcal{G}, \mathbb{F}_{p}\right)^{*} \simeq \bigwedge^{2}\left(\mathcal{G}_{1}{ }^{*}\right)^{*} \simeq \bigwedge^{2}\left(\mathcal{G}_{1}\right) .
$$

Moreover, by class field theory, the finiteness of ray class groups and the fact that $S$ is prime to $p$ imply that the pro- $p$-group $\mathcal{G}^{a b}$ is finite. The Theorem will be established once we take note of the following well-known lemma:

Lemma 8. Let $M$ be a finite $\Delta$-module of order a power of $p$, where $\Delta$ is a finite group of order prime to $p$. Then:

$$
M[p] \simeq_{\mathbb{F}_{p}[\Delta]} M / M^{p} .
$$

$\diamond$

2.3. First applications. When $\Delta$ is abelian, the character of $\bigwedge^{2}\left(\mathcal{G}_{1}\right)$ is easy to estimate (thanks to section 2.1):

Corollary 9. Let $\mathbf{K} / \mathbf{F}$ be a finite abelian extension of number fields of degree prime to $p$ and with group $\Delta$. Let $\mathbf{L} / \mathbf{K}$ be a Galois pro-p-extension with group $\mathcal{G}$ such that:

(i) $\mathbf{L} \subset \mathbf{K}_{S}$; (ii) $\mathbf{L} / \mathbf{F}$ is Galois.

Let $\chi\left(\mathcal{G}_{1}\right)=\sum_{\varphi} \alpha_{\varphi} \varphi$ be the decomposition of $\mathcal{G}_{1}$ as $\Delta$-module.

If

$$
\sum_{\varphi} \alpha_{\varphi}\left(\alpha_{\varphi}-1\right) / 2 \cdot \varphi^{2}+1 / 2 \sum_{\varphi \neq \varphi^{\prime}} \alpha_{\varphi} \alpha_{\varphi^{\prime}} \varphi \varphi^{\prime}<\sum_{\varphi} \alpha_{\varphi} \varphi,
$$

then $\mathcal{G}$ is not uniformly powerful.

Example 10. Suppose that $p>2$ and that $\Delta$ is the Klein 4-group. Let $\varphi_{i}, i=1,2,3$ be its three non-trivial irreducible characters of order 2 . If $\chi\left(\mathcal{G}_{1}\right)=\alpha \varphi_{1}+\beta \varphi_{2}$, then $\mathcal{G}$ is not uniformly powerful.

More generally, the computation of the character of $\bigwedge^{2}\left(\mathcal{G}_{1}\right)$ can be done thanks to the following formula: Let $N$ be a $\mathbb{Q}_{p}[\Delta]$-module. Fix a $\mathbb{Q}_{p}$-basis of $N$ and let $\rho$ be the representation of $\Delta$ associated to $N$. Then, for all $g \in \Delta$,

$$
\chi\left(\bigwedge^{2}(N)\right)(g)=\frac{1}{2}\left(\operatorname{Tr}\left(\rho^{2}(g)\right)-(\operatorname{Tr}(\rho(g)))^{2}\right) .
$$


In fact, thanks to the isomorphism $\bigwedge^{2}\left(N \oplus N^{\prime}\right) \simeq \bigwedge^{2} N \oplus N \otimes N^{\prime} \oplus \bigwedge^{2} N^{\prime}$, one needs to know only the computation of $\Lambda^{2} N$ for an absolutely irreducible $\Delta$-module $N$.

Corollary 11. Let $\mathbf{K} / \mathbf{F}$ be a finite extension of number fields of degree prime to $p$ and with group $\Delta$. Let $\mathbf{L} / \mathbf{K}$ be a Galois pro-p-extension with group $\mathcal{G}$ such that: (i) $\mathbf{L} \subset \mathbf{K}_{S}$; (ii) $\mathbf{L} / \mathbf{F}$ is Galois. Suppose that $\Delta=\mathrm{Sym}_{d}$ is the symmetric group on $d$ letters $(d>3,(d !, p)=1)$. Suppose moreover that $\mathcal{G}_{1}$ is of $p$-rank $d$ and the action of $\Delta$ on $\mathcal{G}_{1}$ is the permutation action on a set of d letters. In others words, $\chi\left(\mathcal{G}_{1}\right)=\mathbf{1}+\varphi$, where $\varphi$ is the character of the standard representation of $\operatorname{Sym}_{d}$ and where 1 is the trivial character. Then the pro-p-group $\mathcal{G}$ is not uniformly powerful.

Proof. The representation $\varphi$ is absolutely irreducible, and moreover $\Lambda^{2}(\varphi)$ is still irreducible (see for example [4], §3). Then $\bigwedge^{2}\left(\mathcal{G}_{1}\right)=\varphi+\bigwedge^{2}(\varphi)$ does not contain $\mathbf{1}$, and so, by Theorem $1, \mathcal{G}$ is not uniformly powerful. $\diamond$

\subsection{The cyclic case.}

One has to compare all of our results with the following result due to Boston:

Theorem 12 (Boston). Let $\mathbf{K} / \mathbf{F}$ be a cyclic extension of prime order $l \neq p$. Let $\mathbf{L} / \mathbf{F}$ be an unramified pro-p-extension such that $\mathbf{L} / \mathbf{F}$ is Galois. If the p-class group of $\mathbf{F}$ is trivial then $\mathcal{G}$ is not uniformly powerful.

In other words, when $\Delta$ is cyclic of prime order $\neq p$, Boston has proved that the representation of $\mathcal{G}_{1}$ necessarily contains the trivial representation.

Suppose that $\Delta$ is generated by $\sigma$ of order $n$ prime to $p$. The absolutely irreducible characters $\varphi_{i}$ of $\mathbb{Q}_{p}[\Delta]$ are of degree 1 defined by: $\sigma \cdot \zeta_{n}=\zeta_{n}^{\varphi_{i}(\sigma)}$, where $\zeta_{n}$ is a primitive $n$th root of unity and where $\varphi_{i}(\sigma) \in \mathbb{Z} / n \mathbb{Z}$.

Let $M$ be an $\mathbb{F}_{p}[\Delta]$-module, and let

$$
A(M)=\left\{\left\langle\varphi_{i}, \chi(M)\right\rangle \varphi_{i}(\sigma), i=0, \cdots, n-1\right\}
$$

be the multiset of elements of $\mathbb{Z} / n \mathbb{Z}$ corresponding to the decompositon of the $\Delta$ module $M($ see $\S 2.1)$.

Definition 13. If $A=\left\{a_{0}, \cdots, a_{s}\right\}$ is a multiset of elements of $\mathbb{Z} / n \mathbb{Z}$, we denote the multiset $\left\{a_{i}+a_{j}, i<j\right\}$ of $\mathbb{Z} / n \mathbb{Z}$ by $\bigwedge^{2} A$.

With the properties developed in section $\S 2.1$, one deduces easily the following proposition: 
Proposition 14. Suppose $M$ is an $\mathbb{F}_{p}[\Delta]$-module and let $A(M)$ be the multiset associated to the $\Delta$-action on $M$. Then $\bigwedge^{2} A(M)$ is the multiset associated to the $\Delta$-module $\bigwedge^{2}(M)$.

Hence, as corollary of Theorem 1, one obtains:

Corollary 15. Let $A\left(\mathcal{G}_{1}\right)$ be the multiset of $\mathbb{Z} / n \mathbb{Z}$ associated to the $\Delta$-decomposition of $\mathcal{G}_{1}$. If the multiset $A\left(\mathcal{G}_{1}\right)$ is not contained in $\bigwedge^{2} A\left(\mathcal{G}_{1}\right)$, then $\mathcal{G}$ is not uniformly powerful.

When $n$ is a prime number $l$, the condition of this corollary does not necessarily imply that 0 is in $A\left(\mathcal{G}_{1}\right)$. (I thank Y. Bilu for this remark). In other words, it does not imply Boston's theorem 12.

Corollary 16. Suppose that $\Delta$ is of prime order $l \neq p$. Let $A\left(\mathcal{G}_{1}\right)=\left\{a_{0}, \cdots, a_{s}\right\}$ be the multiset associated to $\mathcal{G}_{1}$ coming from the action of $\Delta$. Suppose that: $\forall i \neq j$, $a_{i}+a_{j} \neq 0$. Then, $\mathcal{G}$ is not uniformly powerful.

Proof. With these conditions, 0 is not in $\bigwedge^{2} A\left(\mathcal{G}_{1}\right)$ and so Boston's theorem gives the result. $\diamond$

Remark 17. The conditions of this corollary should be compared to the conditions of $[8]$.

2.5. The CM-case. In this section we assume $p>2$. Before giving the result that we need, let us fix some notation. For a finite extension $\mathbf{L} / \mathbf{K}$ of number fields, put

$$
\mathcal{E}(S, \mathbf{L} / \mathbf{K})=\mathcal{E}_{\mathbf{K}, S} /\left(\mathcal{E}_{\mathbf{K}, S} \cap N_{\mathbf{L} / \mathbf{K}}\left(\mathcal{L}_{S}\right) \cdot \mathcal{N}(\mathbf{L} / \mathbf{K})^{p}\right)
$$

where

- $\mathcal{E}_{\mathbf{K}, S}=\left\{x \in \mathbb{Z}_{p} \otimes E_{\mathbf{K}}, x \equiv 1 \bmod v, \forall v \in S\right\}, E_{\mathbf{K}}$ being the group of units of $\mathbf{K}$

- $\mathcal{L}_{S}=\left\{x \in \mathbb{Z}_{p} \otimes \mathbf{L}^{\times}, x \equiv 1 \bmod v, \forall v \in S\right\}$

- $\mathcal{N}(\mathbf{L} / \mathbf{K})=\left\{x \in \mathbb{Z}_{p} \otimes \mathbf{K}^{\times}, x \equiv 1 \bmod v, \forall v \in S\right\} \cap \mathcal{N}_{\text {loc }}$, where $\mathcal{N}_{\text {loc }}$ is the group of elements of $\mathbf{K}^{\times}$that are local norms at every place of $\mathbf{K}$

- $\operatorname{Tor}\left(\mathcal{E}_{\mathbf{K}, S}\right)$ the group of the $p$ th roots of unity in $\mathcal{E}_{\mathbf{K}, S}$. In particular, if $S$ is not empty, $\operatorname{Tor}\left(\mathcal{E}_{\mathbf{K}, S}\right)=\{1\}$.

In the appendix of his book [6], Gras computes the relations of the group $\mathcal{G}_{S}$. His strategy is to study the inflation map Inf:

$$
H^{2}\left(\mathcal{G}_{S} / H, \mathbb{F}_{p}\right) \stackrel{\operatorname{Inf}}{\longrightarrow} H^{2}\left(\mathcal{G}_{S}, \mathbb{F}_{p}\right),
$$


where $H$ is a small open subgroup of $\mathcal{G}_{S}$. He obtains:

Proposition 18 (Gras, [6], Appendix, theorem 3.7). For a sufficiently large finite extension $\mathbf{L} / \mathbf{K}$ inside $\mathbf{K}_{S} / \mathbf{K}$, the following sequence is exact:

$$
1 \longrightarrow \mathcal{E}(S, \mathbf{L} / \mathbf{K}) \longrightarrow H_{2}\left(\mathcal{G}_{S}, \mathbb{F}_{p}\right) \longrightarrow \mathcal{G}_{S}^{a b}[p] \longrightarrow 1 \text {. }
$$

Now let $\mathbf{K} / \mathbf{F}$ be a finite Galois extension of degree prime to $p$ and with Galois group $\Delta$. Let $S$ be a finite set of places of $\mathbf{K}$ prime to $p$. Assume moreover that $S$ is $\Delta$-stable. Then $\mathbf{K}_{S} / \mathbf{F}$ is a Galois extension. Hence in the above proposition, we can assume that $\mathbf{L} / \mathbf{F}$ is a Galois extension. In this case, the short sequence of Proposition 18 is a sequence of $\Delta$-modules.

Lemma 19. The character of the $\Delta$-module $\mathcal{E}(S, \mathbf{L} / \mathbf{K})$ is less than

$$
-\mathbf{1}+\chi\left(\operatorname{Tor}\left(\mathcal{E}_{\mathbf{K}, S}\right)\right)+\sum_{v \in P l_{\infty}} \operatorname{Ind}_{D_{v}}^{G} \mathbf{1},
$$

where $P l_{\infty}$ is the set of archimedean places of $\mathbf{F}, D_{v}$ is the decomposition group of $v$ in $\mathbf{K} / \mathbf{F}$ and $\mathbf{1}$ is the trivial character.

Proof. First note that $\mathcal{E}(\mathbf{K}, S) \subset \mathcal{N}_{\text {loc }}$ (for the archimedean places, recall that we have assumed that $p>2)$. Then the group $\mathcal{E}(S, \mathbf{L} / \mathbf{K})$ is a quotient of $\mathcal{E}_{\mathbf{K}, S} / \mathcal{E}_{\mathbf{K}, S}^{p}$. Now, the group $\mathcal{E}_{\mathbf{K}, S}$ is of finite index in $\mathbb{Z}_{p} \otimes E_{\mathbf{K}}$. Hence, in the semi-simple case, the character of $\mathcal{E}_{\mathbf{K}, S} / \mathcal{E}_{\mathbf{K}, S}^{p}$ can be computed by the Dirichlet map, and, up to $p$ th roots of unity, it is the same as the group of units $E_{\mathbf{K}}$ of $\mathbf{K}$ (see [7] §6).

Now we specialize to the situation where $\Delta$ is of order 2 .

Theorem 20. Assume $p>2$. Let $\mathbf{K} / \mathbf{F}$ be a quadratic extension. Assume that $S$ is $\operatorname{Gal}(\mathbf{K} / \mathbf{F})$-stable. Let $t$ be the number of archimedean places of $\mathbf{F}$ which split completely in $\mathbf{K} / \mathbf{F}$. If $S$ is empty and, $\mathbf{K}$ contains $\mu_{p}$ but $\mathbf{F}$ does not, let $\delta_{p}(S)=1$, otherwise, let $\delta_{p}(S)=0$. Let

$$
d\left(\mathcal{G}_{S}\right)=d^{+}+d^{-}
$$

be the decomposition of the generator rank of $\mathcal{G}_{S}$ according to the action of $\mathrm{Gal}(\mathbf{K} / \mathbf{F})$. If

$$
d^{+} d^{-}>d^{-}+t+\delta_{p}(S)
$$

then $\mathcal{G}_{S}$ is not uniformly powerful.

Proof. By Proposition 18, the rank of the minus part of $H_{2}\left(\mathcal{G}_{S}, \mathbb{F}_{p}\right)$ is less than $d^{-}$ plus the rank of the minus part of $\mathcal{E}(S, \mathbf{L} / \mathbf{K})$. By Lemma 19, the minus part of $\mathcal{E}(S, \mathbf{L} / \mathbf{K})$ is of $p$-rank less than $\delta_{p}(S)+t$. Then, the minus part of $H_{2}\left(\mathcal{G}_{S}, \mathbb{F}_{p}\right)$ is of $p$-rank less than $d^{-}+\delta_{p}(S)+t$. 
Suppose now that $\mathcal{G}_{S}$ is uniformly powerful. Then $\bigwedge^{2}\left(\left(\mathcal{G}_{S}\right)_{1}\right)$ is isomorphic to $H_{2}\left(\mathcal{G}_{S}, \mathbb{F}_{p}\right)$. Moreover, the minus part of $\bigwedge^{2}\left(\left(\mathcal{G}_{S}\right)_{1}\right)$ is exactly of rank $d^{+} d^{-}$and then in this case the minus part of $H_{2}\left(\mathcal{G}_{S}, \mathbb{F}_{p}\right)$ is of rank $d^{+} d^{-}$which contradicts the initial inequality. $\diamond$

As corollary, one find the result of Wingberg [12] given in the introduction:

Corollary 21 (The CM-case). Assume $p>2$. Let $\mathbf{K}$ be a CM-field with maximal totally real subfiel $\mathbf{F}$. Assume that $S$ is $\operatorname{Gal}(\mathbf{K} / \mathbf{F})$-stable. If $d^{+} d^{-}>d^{-}+\delta_{p}(S)$, then $\mathcal{G}_{S}$ is not uniformly powerful.

Proof. In this case $t=0 . \diamond$

\section{Acknowledgements}

The ideas leading to this article were first developed during my visit to the University of Massachusetts, Amherst, in January 2005. I thank Farshid Hajir for his help and his encouragement. I also thank Thong Nguyen Quang Do and Nigel Boston for their interest in this work.

\section{References}

[1] N. Boston, Some cases of the Fontaine-Mazur conjecture, J. Number Theory 42 (1992), 285-291.

[2] N. Boston, Some cases of the Fontaine-Mazur conjecture II, J. Number Theory 75 (1999), 161-169.

[3] J.D. Dixon, M.P.F. Du Sautoy, A. Mann and D. Segal, Analytic Pro-p-Groups, 2nd Edition, Cambridge studies in advanced math. 61, Cambridge Univ. Press, 1999.

[4] W. Fulton and J. Harris, Representation Theory, GTM 129, 1991.

[5] J.-M. Fontaine et B. Mazur, Geometric Galois representations, Elliptic curves, modular forms and Fermat's last theorem, Internat. Press, Cambridge, MA, 1995.

[6] G. Gras, Class field Theory, from theory to practice, SMM, Springer, 2005.

[7] G. Gras, Théorèmes de réflexion, J. Th. des Nombres de Bordeaux 10 fasc. 2 (1998), 399-499.

[8] J. B. Holden, On the Fontaine-Mazur conjecture for number fields and an analogue for functions fields, J. Number Theory 81 (2000), 16-47.

[9] M. Lazard, Groupes analytiques p-adiques, IHES publ. math. 26 (1965).

[10] J.-P. Serre, Linear representations of finite groups, GTM 42, 1977.

[11] P. Symonds, T. Weigel, Cohomology of $p$-adic analytic groups, in "New horizons in pro- $p$ groups", M. du Sautoy, D. Segal, A. Shalev, Progress in Math. 184, 2000.

[12] K. Wingberg, On the Fontaine-Mazur conjecture for CM-fields, Compositio Math. 131 (2002), 341-354.

Department of Mathematics/Computer Science, University Toulouse 2, 5, Allées Antonio-Machado, 31058 Toulouse Cedex 9

E-mail address: maire@univ-tlse2.fr 